\title{
La déterritorialisation des multi-nationales : firmes globales et firmes-réseaux
}

\section{Wladimir Andreff}

\section{(2) OpenEdition \\ 1 Journals}

Édition électronique

URL : http://journals.openedition.org/conflits/161

DOI : $10.4000 /$ conflits. 161

ISSN : $1777-5345$

Éditeur :

CCLS - Centre d'études sur les conflits lilberté et sécurité, L'Harmattan

Édition imprimée

Date de publication : 15 mai 1996

ISSN : 1157-996X

Référence électronique

Wladimir Andreff, «La déterritorialisation des multi-nationales : firmes globales et firmes-réseaux », Cultures \& Conflits [En ligne], 21-22 | printemps-été 1996, mis en ligne le 15 mars 2006, consulté le 30 mars 2021. URL : http://journals.openedition.org/conflits/161 ; DOI : https://doi.org/10.4000/conflits 161

Ce document a été généré automatiquement le 30 mars 2021.

Creative Commons License 


\title{
La déterritorialisation des multi- nationales : firmes globales et firmes-réseaux
}

\author{
Wladimir Andreff
}

Depuis la fin des années soixante-dix, les firmes multinationales (MN) ont développé des stratégies dont l'un des effets majeurs - par rapport à la thématique de ce colloque - a été de les émanciper de façon croissante des contraintes de localisation sur un territoire donné, national, régional ou local. Cette déterritorialisation des MN est passée par plusieurs phases, pas seulement au sens chronologique, mais aussi du point de vue des stratégies menées par ces firmes. Par conséquent, en une date donnée coexistent des MN ayant atteint des degrés différents de déterritorialisation de leurs activités économiques. La première stratégie concernée par notre analyse est connue sous le nom de délocalisation des unités de production des MN. Cette première forme d'émancipation des contraintes de production sur un territoire national d'origine, apparue dès la fin des années soixante, est une sorte "d'ancêtre " (toujours vivant) des modalités plus sophistiquées de déterritorialisation qui ont pris corps à partir des années quatre-vingt. Celles-ci consistent en ce que l'on appelle les nouvelles formes

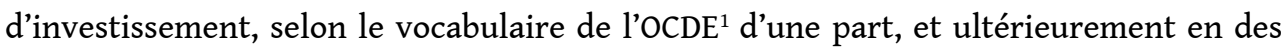
alliances stratégiques entre les sociétés-mères de MN d'origine nationale différente. L'effet commun de ces deux nouvelles formules d'internationalisation des firmes, est de leur permettre d'être présentes dans des pays étrangers, et d'y développer leurs activités économiques sans procéder à un investissement direct à l'étranger (et donc avec une mise de fonds limités, voire presque nulle dans certains cas). Ces nouvelles formes de pénétration des économies étrangères ont résulté, au moins en partie, des contraintes imposées aux MN par des pouvoirs économiques locaux s'exerçant dans les pays d'accueil de leurs filiales (basées sur l'investissement direct), en l'occurrence le pouvoir réglementaire de l'État ${ }^{2}$ et, dans une moindre mesure, le pouvoir syndical local, voire le poids de l'opinion publique ${ }^{3}$. Dans le courant des années quatre-vingt, parmi les firmes-réseaux résultant des stratégies qui viennent d'être évoquées, un certain nombre de MN s'est engagé dans une stratégie dite globale, basée sur une attitude 
extrêmement flexible vis-à-vis de la localisation territoriale des activités. Ces MN globales arbitrent entre n'importe quelle localisation, en tout point du globe (de l'économie mondiale), et en sont d'autant plus capables qu'elles recourent de plus en plus aux technologies modernes de l'information et de communication et qu'elles “ financiarisent " leurs activités. La catégorie la plus déterritorialisée de MN se compose d'une cinquantaine de banques les plus multinationales du monde (les BMN), toutes issues de pays de la Triade, et opérant 24 heures sur 24 sur un marché financier devenu totalement mondial. On peut sans doute parler dans ce cas de MN a-territoriales, la localisation de la banque-mère ou des filiales devenant quelconque du fait de l'existence de zones bancaires off shore, parfois situées dans des paradis fiscaux, et plus récemment $\mathrm{du}$ fait que le marché financier est désormais devenu totalement planétaire. Si les stratégies évoquées ci-dessus, permettent probablement de donner corps à l'idée d'un “ international sans territoire ", celles des BMN valident le thème d'un " mondial ubiquiste ", où la référence au territoire n'a plus grand sens, puisque ces banques peuvent développer leurs activités partout à la fois sans être localisées nulle part ou presque. La notion de réseau (de succursales et filiales) est devenue aujourd'hui extrêmement secondaire dans la stratégie des BMN, comparée à celle de mondialisation de leurs opérations financières.

Déterritorialisation ou exterritorialisation des firmes Il ne s'agit pas ici de sacrifier à un quelconque goût du néologisme, mais plutôt de trouver un nom au phénomène nouveau qui résulte des plus récentes stratégies des MN vis-à-vis du territoire. Commençons par adopter une définition pas trop contraignante de la notion de territoire. On conviendra que, dans la suite, le territoire est une étendue de surface terrestre, éventuellement discontinue (comme le Pakistan naguère), sur laquelle s'exerce une autorité, une juridiction, autrement dit un espace délimité par des règles de compétence. Du point de vue de la firme, le territoire est une variable exogène dont elle tient compte dans l'élaboration de ses stratégies économiques et financières, que cette variable soit très importante, comme autrefois, ou que son influence se soit réduite, parfois jusqu'à néant, comme aujourd'hui. On peut alors définir la territorialité d'une firme par son appartenance, son rattachement à un territoire. Ce qui revient à dire qu'elle suit les règles et les lois qui s'appliquent à ce territoire (elle y paie des impôts, elle y poursuit d'autres acteurs économiques en justice ou elle y est poursuivie, etc.) et elle s'y trouve soumise à une autorité (réglementaire, juridique, parfois politique) en cas d'infraction. A un moindre degré, elle est influencée dans ses stratégies par les orientations de politique économique (régionale, locale, nationale) qu'énoncent les diverses autorités compétentes sur le territoire considéré. L'existence de la territorialité d'une firme donne un sens à l'expression " une MN américaine ". Le fait d'être une MN signifie que cette firme a réalisé des investissements directs à l'étranger (IDE) et y développe une partie de ses activités et de ses stratégies. Le fait de pouvoir dire qu'elle est américaine désigne la réalité selon laquelle cette firme est (encore) rattachée au territoire des États-Unis et que de ce rattachement dépend en partie sa stratégie d'ensemble. Par exemple, on peut supposer que dans ses activités économiques réalisées aux États-Unis, la MN américaine se comportera en " bon citoyen " (paiera ses impôts, etc.). Et dans ses opérations à l'étranger, elle tiendra un large compte des intérêts des États-Unis, bien qu'alors elle ne soit plus directement impliquée sur le territoire américain. Par exemple, elle ne spéculera pas contre le dollar américain (ou moins que les MN françaises, japonaises, allemandes, etc., rattachées à d'autres territoires nationaux), elle fera de l'espionnage industriel en 
faveur d'intérêts américains plutôt que japonais, allemands, etc., ou encore elle rapatriera souvent ses profits aux États-Unis pour pouvoir servir des dividendes à ses actionnaires en majorité américains. Le phénomène nouveau, depuis une quinzaine d'années, est que, en nombre croissant, les MN perdent de plus en plus les attributs que nous venons d'associer à la notion de territorialité. En introduisant notre propos, ce phénomène a été nommé déterritorialisation. Il existe dans la littérature économique, naissante et récente, sur ce sujet une expression concurrente : "l'exterritorialisation " des entreprises ${ }^{4}{ }^{5}$. Celle-ci est alors comme " la résultante des comportements dynamiques, initiés ou subis, qui tendent à modifier la nature ou (et) l'intensité du rattachement de l'entreprise à son ensemble territorial de référence ". Et encore : “ l'exterritorialisation est un mouvement (...) qui correspond au dépassement par l'entreprise de tel ou tel ensemble territorial ". C'est le processus par lequel le lien qui rattachait l'espace économique de la firme à un territoire se trouve rompu. Au fond, “ l'exterritorialisation " désigne la même tendance que ce que nous nommons déterritorialisation, à condition de préciser que les entreprises que nous avons en vue sont déjà des $\mathrm{MN}$ et que leurs comportements vis-à-vis du territoire sont plus souvent initiés que subis. Cela justifie-t-il un néologisme supplémentaire? Assurément, mais pour une raison méthodologique plutôt qu'en fonction d'une différence entre le processus décrit par " déterritorialisation " et par " exterritorialisation ". La dernière expression analyse la perte de territorialité des entreprises vue à partir de leur territoire d'origine ou de référence, à partir du territoire breton dans l'étude citée ${ }^{6}$. Notre approche est une analyse symétrique de la précédente abordant la perte de territorialité des $\mathrm{MN}$ à partir des stratégies qu'elles mettent en oeuvre pour s'émanciper de leur territoire d'origine, puis de tout autre territoire à la limite, ou encore pour rendre la variable exogène " territoire " de plus en plus secondaire ou négligeable, en tout cas de moins en moins contraignante, dans leurs stratégies. Ceci est exactement le sens adopté ici de la notion de déterritorialisation; le même phénomène saisi à partir d'un territoire de référence est l'exterritorialisation. Une autre différence est ce qui ne peut être appréhendé dans une étude de cas sur une région, à savoir que la déterritorialisation est une tendance longue, dont les prémices apparaissent vers la fin des années soixante, et une tendance lourde, concernant un nombre croissant de MN dont le poids dans l'économie mondiale est significatif.

Les multinationales engluées dans le territoire L'IDE s'effectuait habituellement par l'investissement d'un capital dans la propriété d'actifs réels pour implanter une filiale à l'étranger (greenfield investment) ou pour prendre le contrôle d'une entreprise étrangère existante. L'histoire du développement des $\mathrm{MN}$ est, pendant un siècle (de 1870 environ aux années soixante de ce siècle), celle de l'expansion de l'IDE. Pendant cette période, deux stratégies furent successivement adoptées par les MN. La première, dominante jusqu'au début du XXe siècle, était une stratégie d'approvisionnement. Les implantations étaient réalisées à l'étranger pour approvisionner la société mère ou son territoire d'origine à partir de plantations, d'activités extractives et de comptoirs de commerce délocalisés près des ressources naturelles des colonies. Cette stratégie des MN primaires ${ }^{7}$ est encore répandue dans les secteurs miniers et énergétiques et dans les activités métallurgiques qui en dépendent pour obtenir leurs intrants en matières premières. Dans une telle stratégie, la MN est évidemment dépendante du territoire, de ses richesses naturelles et de son sous-sol. La localisation importe au plus haut point. Les autorités politiques et réglementaires, nationales ou locales, sont dans un rapport de force favorable vis-à-vis des MN d'une certaine façon " prisonnières" des territoires 
qui les accueillent; les autorités peuvent user de menaces ou de mesures de rétorsion (pouvant aller jusqu'à la nationalisation de la filiale locale d'une MN). Au XXe siècle, et surtout jusqu'aux années soixante, sont apparues des MN à stratégie de marchés dont les IDE prolongent l'activité d'exportation par une production sur le lieu même de leurs marchés étrangers. Leurs filiales-relais produisent les mêmes produits que ceux de la société mère auxquels ils se substituent et importent des intrants de leur territoire d'origine. Cette stratégie correspond à ce que Porter 9 désigne par stratégie " multi-pays " (multidomestic), donc au sens propre multinationale. Ici encore, les MN sont assez dépendantes de leur territoire d'implantation à l'étranger, mais moins que les MN primaires. Elles sont dépendantes économiquement de la demande existant sur les marchés desservis par les filiales-relais et, également, des interventions de politique économique des États des pays d'accueil touchant les flux de commerce extérieur (droits de douane sur les intrants importés par les filiales) et intérieur (politique des prix et des revenus) ou la régulation de l'économie (fiscalité locale par exemple). L'expression " engluées dans le territoire " de leurs pays d'accueil est sans doute un peu forte. Cette image rend cependant bien compte de la perception des dirigeants de MN lorsqu'ils furent en butte, principalement dans les pays en développement (PVD), à des politiques restrictives vis-à-vis de leurs filiales et de leur IDE, pendant les années soixante et jusque vers 1976-1977. Ces politiques restrictives, décrites ailleurs ${ }^{10}$, consistaient en l'interdiction de l'IDE dans certains secteurs du pays d'accueil, en un plafonnement des profits rapatriés et des redevances technologiques, en des contraintes de performance (exportation, valeur ajoutée locale, emploi d'autochtones, etc.), en un contrôle des changes, voire même en des politiques "d'indigénisation " du capital et, dans le pire des cas (du point de vue des MN), en la nationalisation de leurs filiales locales. De 1960 à 1976, 1369 filiales de MN ont été nationalisées dans les PVD, contre 47 de 1977 à 1985, et quasiment aucune ultérieurement. Entre temps, ayant le sentiment d'être devenus trop dépendantes des territoires de localisation de leurs filiales et des pouvoirs s'y exerçant, les MN ont mis en place de nouvelles stratégies accroissant progressivement leur déterritorialisation.

La délocalisation de la production: la première tentative des multinationales pour s'émanciper des contraintes territoriales . Les IDE tirent parti de coûts de production (en capital, en intrants, en salaires) plus faibles dans les pays hôtes et d'économies d'échelle dues à la forte spécialisation de filiales-ateliers. Celles-ci produisent les composants et intrants des produits de la société mère et les exportent vers le territoire d'origine ou vers des filiales localisées dans des territoires tiers. Ces MN amorcent l'intégration internationale de leur processus de production d'un même produit final : les chaînes de montage de la Ford-Escort, en Allemagne et en Angleterre, recevaient freins et pneus de filiales belges, vitres et autoradios du Canada, batteries et rétroviseurs d'Espagne, poussoirs hydrauliques des États-Unis, carters, alternateurs et ventilateurs de France, démarreurs et roulements du Japon ${ }^{11}$. La stratégie de rationalisation de la production a été adoptée par les MN dont les opérations de production sont segmentables, se prêtant à une décomposition internationale du processus productif (DIPP) ${ }^{12}$. Plus un produit est complexe, plus il contient de composants qui peuvent être fabriqués de façon autonome les uns des autres, plus il offre de possibilités de DIPP, et plus la MN peut associer deux décisions : celle de segmenter (séparer les unes des autres) les opérations de production préalables à l'assemblage du produit final d'une part, et celle de délocaliser certaines de ces opérations dans divers pays hôtes d'autre part. Les activités de production disjointes 
dans l'espace peuvent être localisées librement soit dans des filiales étrangères, soit par sous-traitance ou coopération internationale et approvisionnement à l'étranger, et ce d'autant plus que les coûts de transport baissent continûment. Tous les stades du processus productif ne présentent pas la même intensité en capital technologique (machines spécialisées), en main-d'oeuvre peu qualifiée, en énergie ou en savoir-faire (qualifications). La MN arbitre donc entre les territoires d'accueil potentiels en fonction de leurs avantages comparés pour chaque opération de production. Un effet de la DIPP est la perte d'autonomie et la déstructuration partielle des systèmes productifs nationaux, dont certaines industries passent dans la sphère de décision des $\mathrm{MN}$; c'est la contrepartie logique de l'intégration internationale de la production amorcée dès la fin des années soixante ${ }^{13}$. C'est également la preuve d'une émancipation partielle des MN vis-à-vis des contraintes territoriales. Dans les stratégies antérieures, ce sont les pays d'accueil qui étaient le plus souvent en situation de mettre les MN en concurrence pour une implantation sur leur territoire. Avec les stratégies de délocalisation, ce sont les MN qui se mettent de plus en plus en situation d'arbitrer entre les localisations de plusieurs pays hôtes potentiels. Par conséquent, la flexibilité accrue de localisation des MN offerte par la DIPP se traduit par des délocalisations et des relocalisations de filiales, redoutées par les territoires qui les subissent. La difficulté est que, si une MN délocalise, d'autres MN sont contraintes à le faire aussi pour rester compétitives dans un cadre de concurrence oligopolistique; tel fut le cas des industries de l'habillement, de la chaussure, des articles de sport, du jouet, de l'électronique et de l'automobile. Cette flexibilité perdure avec les stratégies des MN globales (cf. note 5) qui ont une conception globale de la localisation arbitrant entre tous les pays et les régions du monde désireux d'attirer leurs IDE en faveur du mieux offrant ${ }^{14}$. A la limite, la menace de délocalisation est utilisée comme moyen de discipliner la main-d'oeuvre, en matière de salaire et de conditions de travai ${ }^{15}$. Quand les MN américaines ont commencé à délocaliser en Asie vers la fin des années soixante, selon les syndicats américains, les délocalisations auraient supprimé près d'un million d'emplois aux États-Unis. Des études postérieures ont montré que, si l'on tient compte aussi des effets indirects des délocalisations, elles ont procuré une création nette d'emplois aux États-Unis. L'effet sur l'emploi de la délocalisation est en fait assez variable suivant les cas d'espèce et il est difficile de trancher ${ }^{16}$

, mais la menace de délocalisation, utilisée par les MN comme une sorte de "chantage" à l'emploi, a assurément un effet disciplinaire sur la main-d'oeuvre, syndiquée ou non, résidant sur un territoire donné.

Les firmes-réseaux: une présence moins risquée dans les territoires nationaux ou locaux Les statistiques de l'IDE sous-évaluent le poids des MN dans l'économie mondiale. Cette sous-évaluation est en partie due à la non prise en compte des relations développées par les MN sans engager de capitaux (ou peu), nommées les nouvelles formes d'investissement (NFI). Il s'agit des filiales étrangères où la MN possède moins de $50 \%$ du capital, des accords de licence, de l'assistance technique, du franchisage, de la sous-traitance internationale, des accords de partage de la production, de la coopération industrielle, des contrats de gestion ou de service, des livraisons d'usine clé en main, produit en main ou marché en main et des accords de cofinancement. Tout en liant les MN au territoire d'accueil sur longue durée, les NFI immobilisent peu ou pas de capital, dissocient la technique et l'expertise de l'élément financier et font jouer un effet de levier au profit des MN (le pays d'accueil doit fournir l'essentiel des capitaux et parfois s'endetter dans ce but). Plus les NFI se sont substituées à des IDE classiques dans 
les PVD, puis dans les pays développés à économie de marché (PDEM) où elles se sont doublées de stratégies d'alliances entre les sociétés mères, plus le lien est devenu lâche entre la MN et le territoire d'accueil, comparé à l'ancien IDE. En réaction notamment aux politiques restrictives ou hostiles des États contrôlant certains territoires d'accueil de leurs activités, les MN ont tendu de plus en plus, à partir du milieu des années soixante-dix, à contrôler des sociétés étrangères sans apport de capital, en devenant leur fournisseur exclusif de technologie ou leur client unique, grâce aux NFI. Quand son processus de production est décomposable, au sens de la DIPP ci-dessus, la MN peut externaliser des opérations de production à la fois hors de la firme et de ses filiales étrangères et hors de son territoire d'origine, dans le cadre de la sous-traitance internationale. Une relation de sous-traitance existe quand une entreprise (soustraitant) intervient pour le compte d'une autre entreprise (donneur d'ordre) dans le processus d'élaboration et de réalisation de l'un de ses produits spécifiques; elle est internationale lorsque les deux partenaires sont localisés dans des pays différents. Elle implique nécessairement l'existence d'un flux d'importation et/ou d'exportation entre les deux entreprises qui, sans avoir les propriétés du commerce captif intra-MN, n'est pas du commerce extérieur traditionnel. Il s'agit de flux donnant lieu à des opérations d'ouvraison (montage, assemblage), de transformation et de réparation de produits. L'accord OEM dit de fabrication d'équipements originaux (original equipment manufacturing arrangement) est une forme de coopération internationale qui s'est développée dans les années quatre-vingt. C'est un contrat à long terme, renouvelable, liant une MN et ses principaux fournisseurs étrangers de composants ou de produits semi-finis, à des prix fixés pour la durée du contrat, avec des délais de livraison spécifiés et des normes de qualité à respecter. La MN intègre les composants dans le produit final qu'elle seule commercialise. L'OEM permet à une MN de s'assurer de son approvisionnement à l'étranger, de se concentrer sur sa production principale et de laisser à ses partenaires la fabrication de produits qui ne sont pas de sa compétence technique directe; c'est le partenaire qui doit s'adapter aux contraintes territoriales locales et non plus la MN (il en va de même avec la sous-traitance). Quant aux partenaires, souvent des PME, l'OEM leur garantit un débouché et de grosses commandes stables de la MN. Les grandes MN, américaines notamment, ont utilisé les accords OEM pour répondre à la concurrence, en coopérant avec des firmes des nouveaux pays industriels à coûts de production plus faibles que les MN japonaises concurrentes. Dans les années quatre-vingt, nombre de MN ont poursuivi leur internationalisation en se recentrant sur leur métier et sur des gammes de produits plus resserrées. Cette intégration internationale jouant sur les synergies et les complémentarités pousse les MN à abandonner les activités éloignées de leur métier de base et à investir dans la haute technologie, ce qu'illustre la stratégie des MN de l'informatique et de la chimie ${ }^{17}$. Cette respécialisation a des effets sur la localisation, en faveur des PDEM, sur la taille des unités de production, en baisse, sur les effectifs employés dans le monde et sur les firmes que les MN décident d'acquérir ou d'absorber dans le même métier. Le recentrage sur l'amont technologique ${ }^{18} \mathrm{se}$ traduit par une concurrence exacerbée au sein des oligopoles en pleine recomposition, devenant mondiaux : à l'horizon de l'an 2000, dans certains secteurs ou filières, il ne devrait plus rester que trois ou quatre grands producteurs ${ }^{19}$. Chacun disposant d'un réseau d'unités de production spécialisées à l'échelle mondiale. Telle est la MN-réseau, articulant des filiales, des NFI et des firmes absorbées au cours de sa croissance externe, le tout sur un espace international. Sur une partie de ce réseau, les autorités des territoires d'accueil 
n'ont plus de prise. On ne peut pas nationaliser une entreprise livrée clé en main à une entreprise locale avec l'espoir d'infléchir la stratégie de la MN (qui n'est pas propriétaire de l'usine qu'elle livre). Comment les autorités locales pourraient-elles contrôler ou nationaliser un accord d'assistance technique ou un accord OEM ? L'émancipation territoriale des MN devient nettement plus prononcée avec les NFI qu'elle ne l'était avec les possibilités de délocalisation de la production. D'autre part, la DIPP s'est développée dans l'état des techniques correspondant au fordisme (processus continu de fabrication, automatisation rigide, machines spécialisées, réponse quantitative plus que qualitative à la demande). Les années quatre-vingt marquent l'épuisement des techniques fordiennes et l'apparition d'une demande personnalisée et versatile, exigeant des gammes de produits renouvelées et une production flexible, en petites séries. Les $\mathrm{MN}$ ont dû s'adapter à ce nouveau paradigme techno-économique et se doter de machines automatiques, programmables et flexibles dans leur utilisation, permettant des économies de variété, au lieu des économies d'échelle du fordisme ${ }^{20}$. Avec l'automatisation flexible, l'information, la connaissance et la RechercheDéveloppement (RD) deviennent les intrants cruciaux de la production et leur utilisation est moins coûteuse quand elle est concentrée dans le même espace de production. Les segments du processus productif sont alors réintégrés en un même lieu, ce qui fait obstacle à la DIPP; quant au caractère intense en technologie de la production, il oriente ce qu'il subsiste de la DIPP vers les PDEM et peu vers les PVD à bas salaires. Ceci est renforcé par la découverte des matériaux nouveaux et les biotechnologies qui se substituent aux ressources naturelles des $\mathrm{PVD}^{21}$. Les connaissances scientifiques et techniques deviennent une sorte de matière première conférant des avantages absolus de coûts très supérieurs à ceux qui reposent sur de bas salaires. Le capital immatériel (logiciel) se substitue de plus en plus au capital matériel (équipement). Ces changements techniques engendrent une recomposition internationale des processus productifs et une relocalisation des activités d'assemblage et de production dans les PDEM, territoire d'origine de la MN ou territoire d'un pays tiers. Déjà partiellement libérées des contraintes de territoire par les possibles délocalisations et par les NFI, les MN-réseaux sont ainsi dotées d'un instrument supplémentaire, la relocalisation ou la menace de relocalisation, pour flexibiliser l'attitude des autorités ou des forces sociales ayant pouvoir sur un territoire quelconque. Ces mutations technologiques, devenues le facteur décisif de leur compétitivité, ont exigé des MN un gros effort de RD, ainsi que son internationalisation depuis la fin des années quatre-vingt ${ }^{22}$. La délocalisation de la RD, l'établissement de laboratoires hors du territoire d'origine, sans rapport avec la délocalisation de la production, est l'indice d'une globalisation technologique, selon l'OCDE, du moins au sein de la Triade. En 1966, la RD totale des MN américaines était délocalisée à 6,5\%, à $13 \%$ en $1989^{23}$. La télématique permet de transmettre les informations relatives aux nouveautés techniques à travers tout le réseau de la MN en temps réel et d'intégrer internationalement les travaux des différents laboratoires. Les flux d'innovations sont à leur tour en partie déterritorialisés. Le cycle de vie des produits nouveaux se raccourcit en proportion directe de la croissance des dépenses de RD, jusqu'à disparaître. D'autant plus que les nouvelles technologies de communication et d'information propagent instantanément la nouveauté à tous les marchés du globe. D'autre part, les innovations réalisées par les MN localisées sur leur territoire remettent en cause les avantages comparatifs acquis dans le passé par les différentes pays participant au commerce mondial ${ }^{24}$. La consolidation des MN-réseaux se parachève par des alliances stratégiques 
entre MN. Celles-ci ont progressé de 41\% entre 1980-1984 et 1985-1989. Les alliances américano-européennes sont plus nombreuses et plus dynamiques que les américanojaponaises, et celles-ci que les nippo-européennes. Ces alliances donnent naissance à des réseaux de MN (têtes de groupe) qui sont elles-mêmes des firmes-réseaux ${ }^{25}$ (réseau articulé de filiales, de sous-traitants, de NFI, de liaisons personnelles, communiquant par télématique). La délimitation entre l'intérieur et l'extérieur de la MN devient floue, dans une situation qui n'est ni une réelle internalisation (hiérarchie au sein d'un groupe), ni une réelle externalisation (marché et contrats entre MN). L'alliance est une position moyenne entre le marché et l'organisation, visant à réduire à la fois les coûts de transaction et les coûts de contrôle ${ }^{26}$. Au sein de ces réseaux d'alliances, les MN sont en même temps concurrentes, pour telle activité ou tel marché, et coalisées pour d'autres, spécialement la $\mathrm{RD}^{27}$. Les frontières de l'oligopole mondial et de l'industrie correspondante s'estompent, atténuant la pertinence d'un raisonnement, et a fortiori de stratégies, menés en termes de territoires locaux ou nationaux. Dans certains cas, l'alliance permet à une $\mathrm{MN}$ de ne pas s'établir sur chaque territoire où elle fait des affaires, donc de réduire sa dépense en capital, et de se faire représenter localement par un allié, et de contourner définitivement les contraintes territoriales. Bien que des alliances interviennent en n'importe quel point de la chaîne de valeur de la MN, elles sont en majorité des accords de coopération à un effort commun de RD dans des activités de haute technologie. Les MN d'un même réseau se répartissent les coûts de plus en plus importants de la $\mathrm{RD}$, en assument les risques ensemble, ainsi que l'obsolescence rapide des nouveaux produits, et partagent les innovations et l'information scientifique ${ }^{28}$. Les alliances sont diversifiées tout au long de la chaîne de valeur de la MN : IBM est allié à Thomson-CSF dans les microprocesseurs, à Microsoft (puis Apple) pour les micro-ordinateurs, à Ricoh pour la distribution d'ordinateurs bas de gamme, à Nippon Steel pour l'intégration des systèmes, à la banque Fuji pour la commercialisation de systèmes financiers, à Toshiba et Siemens dans les mémoires DRAM depuis 1992, accord aussitôt suivi d'une alliance rivale entre NEC et ATT. Le caractère combinatoire des récentes découvertes techniques et la coopération entre MN d'origine industrielle variée donne une nature intersectorielle aux nouvelles technologies. Le coût d'accès à celles-ci pour les firmes qui ne participent pas aux alliances peut devenir prohibitif (barrière à l'entrée) et l'est pour les firmes des PVD. Les oligopoles mondiaux ne disparaissent pas, mais sont plus mouvants et perméables, chaque MN ayant une position contestable au gré des nouvelles alliances; aucune alliance n'est irrévocable. L'oligopole mondial est basé sur une coopération-rivalité où chaque MN coopère avec ses rivaux pour rester compétitive et concurrence ses alliés en s'appuyant sur leur commun accord de coopération. Le type de contrat scellant l'alliance et les secteurs de coopération deviennent incomparablement plus importants que les territoires dans les stratégies des MN. Notons d'ailleurs qu'une alliance n'implique ni un changement de localisation des partenaires, ni nécessairement une création de filiale commune (bien que possible), ni un déplacement de capital ou de production d'un territoire à un autre, par rapport à la situation initiale. On n'ira pas jusqu'à soutenir que les alliances entre MN sont territorialement neutres, mais il faut bien admettre que la préoccupation territoriale est loin d'y être prédominante. Certaines firmes-réseaux se constituent uniquement sur la base de la sous-traitance et de la délocalisation de la production; ce sont en général des MN de taille plus modeste que les parties prenantes aux alliances comme Nike, Reebok, Nintendo. Ainsi, Nike sous-traite $90 \%$ de sa production en Asie et ne se présente pas comme une firme 
industrielle, mais comme spécialiste en conception, innovation et commercialisation; elle devient à la limite une " firme creuse " (hollow corporation) ${ }^{29}$, au moins en matière industrielle.

La "glocalisation " des multinationales globales Les stratégies globales adoptées à la fin des années quatre-vingt par un nombre croissant de MN ont une triple cause: les mutations technologiques; l'adaptation à l'après-DIPP ; la réaction à l'incertitude d'une crise devenue durable et aux risques encourus dans les pays hôtes, surtout les PVD (instabilité économique et politique, nationalisations). Elles furent précédées de la stratégie techno-financière ${ }^{30}$ caractérisée par un glissement de l'IDE vers les NFI, la sous-traitance et les alliances entre MN, par un dégagement des activités de production et un engagement dans la RD, la fourniture de services et la recherche de gains spéculatifs facilitée par la globalisation financière (cf. note 6), par le passage du contrôle du capital et de la filialisation vers la maîtrise d'une activité à l'étranger grâce à la technologie et au financement à partir d'une société mère ou d'une holding localisée si possible en un paradis fiscal. On passe à une véritable globalisation de la stratégie d'une " MN de style nouveau " lorsque, simultanément, elle a les caractéristiques suivantes. La MN a une vision mondiale des marchés et de la concurrence. Elle connaît bien ses rivaux, la mondialisation de la concurrence n'étant pas anonyme et créant une interdépendance entre toutes les MN de l'oligopole ${ }^{31}$. Elle a le pouvoir de contrôler ses opérations dans l'espace de la Triade ${ }^{32}$. Elle se comporte comme un " joueur global " et change radicalement sa façon de travailler, sa survie étant mise en jeu par une concurrence aiguë dans l'oligopole mondial. Elle opère dans des industries à haute technologie et y recherche des actifs porteurs d'innovation sur une échelle globale ${ }^{33}$. Elle localise ses activités là où elles sont les plus rentables, suivant les avantages comparés offerts par les pays, en n'importe quel point du globe de sorte qu'on a pu énoncer l'affreux néologisme de " glocalisation ". Elle a des activités coordonnées à l'aide des technologies de l'information et de production flexible ${ }^{34}$, créant de la valeur ajoutée dans de nombreux pays, et intégrées en une " chaîne de valeur " ${ }_{35}$ internationale, sur une base régionale ou mondiale. Elle organise ses usines et filiales spécialisées en un réseau internationalement intégré ${ }^{36}$ et s'intègre dans un réseau d'alliances avec d'autres $\mathrm{MN}^{37}$. La stratégie globale n'est pas seulement technique et financière ; elle est aussi, profondément, industrielle et commerciale, de marché et de production. Outre les études de cas, cinq indices permettent de repérer les stratégies globales: la centralisation internationale du capital, la structure de groupe prise par les MN, leur traitement de la RD et de la technologie (supra), leurs alliances avec d'autres MN et l'intégration mondiale de leur production. On compte parmi les MN à stratégie globale notamment Ford, General Motors, IBM, HewlettPackard, Texas Instruments, ITT, Xerox, Monsanto, Du Pont, Mobil, Procter \& Gamble, Levi Strauss, Sony, Mazda, Honda, Toyota, Hitachi, Toshiba, Asea Brown Boveri (ABB), Ciba-Geigy, Swissair, Volkswagen, Siemens, Philips, IKEA, Electrolux, ICI, Glaxo, BP, Thomson, Benetton, Hyundai. La centralisation du capital opère par fusion, acquisition ou prise de participation au capital entre deux (plusieurs) firmes. Elle est internationale lorsqu'y interviennent des $\mathrm{MN}$ ou des BMN, ou quand elle réunit les capitaux de firmes de pays différents. Elle est un mode de recomposition des oligopoles et, lorsqu'elle est internationale, elle accroît la pénétration réciproque des marchés intérieurs des PDEM par leurs propres MN. Pour celles-ci, c'est un moyen de faire croître leur part du marché mondial même quand ce dernier n'est pas dynamique. En 1979 et 1980, respectivement 666 et 721 firmes américaines ont été acquises par des $\mathrm{MN}$ étrangères, 
annonçant une nouvelle vague de centralisation qui s'est accélérée dans toute la Triade de 1986 à 1990. Ces cinq années enregistrent une "explosion" des achats de firmes américaines par des MN étrangères, des fusions entre firmes de différents pays de la CEE et, à un moindre degré entre entreprises européennes et firmes tierces nonaméricaines (surtout japonaises). La déréglementation et l'interconnexion des marchés financiers (cf. note 6), la globalisation financière, et la sous-capitalisation boursière des firmes américaines et européennes ont également facilité la récente vague de fusions. Les acquisitions signalent à la fois la dérive financière de certaines MN embarquées dans une stratégie techno-financière et les restructurations requises par les nouvelles technologies, par le démantèlement des conglomérats et par le recentrage ouvrant la voie aux stratégies globales. Deux résultats de la centralisation internationale du capital sont une forte concentration sur les marchés mondiaux et le renforcement des MN structurées en groupes industriels et financiers. Conséquence : la concurrence augmente en intensité entre les MN sur chaque territoire national ; leur coopération, voire collusion, la réduit au niveau de chaque industrie mondiale. Les six industries qui ont vu le plus grand nombre d'acquisitions réalisées dans le monde en $1985-1991^{38}$ sont la chimie, le pétrole, l'agro-alimentaire, les télécommunications, l'électronique et la machine-outil. La résultante des stratégies globales des MN est la formation d'un système productif international intégré par rapport auquel le territoire devient une variable secondaire. La convergence des modes de consommation dans les PDEM - en partie impulsée par la diffusion des produits des MN (Coca Cola, Mc Donald, Nestlé, Unilever, etc.) - et la baisse des coûts de transport se conjuguent à la télématique pour permettre aux MN de mener des stratégies d'intégration de la production et de la distribution à un niveau mondial, continental ou régional (au sens large: Europe de l'Ouest, Asie du Sud-Est). Les produits sont globalement standardisés et, en même temps, différenciés pour s'adapter aux préférences locales. La qualité, la présentation et le service après-vente des produits différenciés sont gérés globalement et, face à une demande versatile, l'emportent sur les considérations de coût de production. Pour être globales et localement adaptées, les MN mettent en place des formes d'organisation nouvelles. Par exemple, une unité localisée dans un pays est responsable de la RD de la MN pour le monde ou un continent entier, une autre unité localisée ailleurs l'étant pour la finance et une troisième pour le marketing; toutes les trois ensemble, elles forment un système de contrôle d'une stratégie unifiée de la MN. Chaque unité, chaque filiale, chaque division de la MN se voit confier des responsabilités qui sont clairement définies comme une partie de la division mondiale du travail interne à la MN. Les relations en réseaux facilitent cette dernière et la rendent très flexible. Le produit offert sur le marché par la MN - parfois nommé " produit-système " - est alors un composé complexe d'intrants, fabriqués dans les localisations les plus diverses (les territoires n'étant bien souvent dans ce contexte qu'une variable d'ajustement ou d'atténuation marginale des coûts), assemblés en pays d'origine ou dans l'un quelconque des pays hôtes, produit destiné à être vendu n'importe où dans le monde. Un tel produit ne s'identifie plus à un label Made in (suit le nom d'un pays) mais à Made in (suit le nom d'une MN). Il est donc complètement déterritorialisé, n'a quasiment plus aucune forme de rattachement à un territoire. Un nombre croissant de MN desservent les marchés mondiaux à partir de réseaux concentrés dans un continent ou une région, ce qui est une étape vers une intégration mondiale encore plus poussée. Dans ce but, les MN globales procèdent à la mise en réseau mondial de leurs activités (global networking), à leur commutation-délocalisation-relocalisation - à l'échelle mondiale 
(global switching) et à la concentration de certaines fonctions (RD, finance, etc.) en des sites sélectionnés dans l'économie mondiale (global focusing) ${ }^{39}$. Par exemple, Swissair a transféré sa comptabilité en 1993 à sa filiale Airline Financial Support Services India, à Bombay, dans laquelle $25 \%$ du capital sont détenus par la firme indienne Tata Consulting Services. Quant aux transactions (achats réciproques de billets) entre Swissair et d'autres compagnies aériennes alliées, elles sont compensées par une unité localisée à Londres. Le logiciel connectant Bombay, Londres et le siège à Zurich a été élaboré par une firme californienne. Ce cas démontre que les stratégies globales commencent à se développer aussi bien dans les services.

La mondialisation ubiquiste des banques multinationales Les services sont devenus le principal secteur d'expansion des MN. L'IDE tertiaire se situe à $85 \%{ }^{40}$ dans les services financiers (banque, assurance, sociétés financières) et le commerce. L'internationalisation des banques et des services financiers en est le domaine le plus avancé. Sur le marché planétaire de la finance, l'ampleur et la rapidité des transactions des BMN propagent des effets incontrôlables (crise de change, krach financier). De la lire au peso mexicain toute monnaie est sous la menace de la spéculation, du CAC 40 au Nikkeï aucun indice boursier n'est à l'abri des choix des investisseurs internationaux, de Wall Street à Zurich aucune place financière n'est imperméable aux alertes récurrentes. La globalisation de l'économie mondiale par les MN est avant tout financière. Tout d'abord, les stratégies techno-financières et globales des MN en ont fait d'importants opérateurs sur les marchés financiers internationaux. D'autre part, chaque MN organise une circulation de fonds interne, une sorte de " marché " financier international intérieur au groupe, de façon à localiser les profits ou le financement des IDE dans tel territoire de son choix. La MN soit crée dans ce but une holding financière, soit crée ou acquiert sa propre banque de groupe : la banque Morhange par Schneider (1987), la BATIF par Thomson (1984), Alfabanque par BSN. Les fonds sont déplacés d'un lieu et d'un territoire à l'autre, au sein de la MN, par des transferts de capitaux entre les filiales et la société mère (ou la holding) : prêts intra-MN, règlements des filiales au groupe ou l'inverse ; par des transferts explicites de revenus, rapatriement des profits, versement de redevances technologiques et d'honoraires par les filiales à la mère ${ }^{41}$; et par des transferts de fonds implicites. Ces derniers sont réalisés par la fixation discrétionnaire des prix de cession interne entre les filiales et la mère, par la rémunération de services fictifs entre elles ou même par de fausses déclarations au sujet de la valeur des produits circulant entre pays au sein de la $\mathrm{MN}^{42}$. Elles peuvent enfin se couvrir à l'aide de nouveaux produits financiers sur les marchés dérivés. Toutes ces opérations financières permettent en outre à la $\mathrm{MN}$ d'emprunter là où les taux d'intérêt sont plus faibles, de diversifier ses sources de financement et de contourner les politiques locales de restriction du crédit ou d'autres réglementations limitatives. Pour de telles opérations, la notion de territoire est quasiment vide de sens, la déterritorialisation presque totale. Les réserves officielles de change des PDEM ne représentent pas plus que le montant quotidien des transactions sur le marché des changes ${ }^{43}$. Les autorités monétaires d'un pays n'ont plus le pouvoir de défendre leur taux de change face à la spéculation et les politiques économiques nationales perdent de leur autonomie et de leur efficacité. La trésorerie cumulée de toutes les MN du monde représente plusieurs fois le montant des réserves monétaires officielles (de l'ensemble des banques centrales), si bien que le déplacement de 1 à $2 \%$ de ces masses financières d'une devise sur une autre est de nature à modifier la parité entre deux monnaies. Le FMI, ni quelque autre autorité monétaire, ne dispose d'un chiffre précis 
quant à leur montant, mais estime que les opérations de change à partir de leur trésorerie sont considérées de façon croissante par les $\mathrm{MN}$ comme des centres de profit. Les MN en tirent un véritable pouvoir monétaire international, auquel s'ajoute celui des BMN. La monnaie cesse d'être l'un des attributs du pouvoir s'exerçant sur un territoire, celui où circule cette monnaie, puisque le territoire de circulation des monnaies convertibles est devenu le globe tout entier (pour sa partie terrestre). Conjointement avec les trésoreries des $\mathrm{MN}$, les opérations des BMN et des grandes sociétés financières non bancaires, telles American Express, Merrill Lynch, Nomura Securities, Morgan Stanley, Salomon Inc., qui sont aussi des MN, forment un seul marché planétaire des changes: les transactions sur le franc, la lire, le yen se font aussi bien et en même temps à Londres, New York, Hong Kong, Bahrein, par téléphone, télex et télématique. Cinquante BMN des pays de la Triade sont les principaux opérateurs sur le marché interbancaire des xeno-devises et sur les marchés internationaux de titres et de produits dérivés. La déréglementation bancaire, sauf dans les PVD, et le décloisonnement entre les marchés des changes, des crédits, des obligations et des actions a accru la concurrence entre les BMN, les sociétés financières et les MN ellesmêmes. Sur un marché des capitaux de plus en plus global, les BMN opèrent 24 heures sur 24 (grâce à la télématique), la mobilité des liquidités est instantanée, les opérations mêlent le court et le long terme et le passage de la finance indirecte (crédits) à la finance directe (titres) est facilitée et accrue. La valeur unitaire des transactions et les masses financières impliquées sont énormes, en particulier sur le marché de gros interbancaire : 187 milliards de dollars de transactions par jour, malgré le netting, sur le marché des changes de Londres en 1991 et 1300 milliards par jour sur le marché planétaire en 1994, selon la BRI. Le volume des opérations de change est ainsi cinquante fois plus important que celui du commerce mondial de biens et services ${ }^{44}$. Le marché des changes est le compartiment du marché aujourd'hui le plus rentable pour les BMN, montrant que les distinctions entre les opérations de change liées aux transactions commerciales et celles réalisées en vue de gains en capital (spéculation pure), tout comme entre l'arbitrage sans risque de change et la recherche risquée de profits spéculatifs, sont devenues très étroites ${ }^{45}$. On parle alors d'économie internationale de spéculation $^{46}$, voire d'économie (mondiale) de casino. Les opérations financières des BMN et des MN sont de moins en moins contraintes par la localisation géographique de leurs réseaux de filiales. A la limite, le territoire ne compte plus. Ces opérations ont produit une intégration des marchés de capitaux internationaux, une globalisation financière de l'économie mondiale. Le volume des dettes entre BMN a été multiplié par 12 entre 1970 et 1990, celui de leurs crédits transnationaux par 32 et celui de leurs dépôts transnationaux par 23. Les prêts bancaires internationaux nets (à des emprunteurs non bancaires) est passé de 7,5\% du commerce mondial en 1964 à 104,6\% en 1991, et de 6,2\% de l'investissement brut mondial à 131,4\% en 1991. La globalisation financière apparaît plus marquée quand on prend en compte les opérations interbancaires internationales qui représentent plus de deux fois le commerce mondial et presque trois fois l'investissement mondial en 1991. En 1992, la taille des marchés financiers mondiaux était estimée à 43.000 milliards de dollars ${ }^{47}$. Les mouvements de capitaux des MN et des BMN deviennent largement autonomes du financement de la production et des échanges, et par conséquent des territoires sur lesquels ils se déroulent. Les marchés des euro-(xeno-) devises et des xeno-crédits à moyen terme ont cédé le pas aux marchés des xeno-titres (obligations, produits dérivés, actions). Les opérations transnationales sur actions et obligations (achats et ventes en gros de titres 
entre résidents et non résidents) s'élèvent de 12\% du PIB en 1982 à 109\% en 1992 aux États-Unis, de $13 \%$ à $91 \%$ en Allemagne, de $8 \%$ à 122\% en France, de 366\% en 1985 à 1017\% en 1991 au Royaume-Uni. Les xeno-obligations sont des obligations émises par un syndicat de BMN dans un pays différent de celui de la monnaie qui leur sert de support et souscrites principalement dans d'autres pays que celui de la monnaie dans laquelle elles sont libellées. Apparues sur le marché en 1976, les xeno-actions sont émises par une MN sur des marchés boursiers autres que son marché national d'origine. La propriété des capitaux cesse, elle aussi, d'être rattachée à un territoire national. Que peut encore signifier l'expression “ une MN américaine " ? Principaux intermédiaires financiers sur les marchés des xeno-crédits, les BMN ont dû se reconvertir vers les transactions sur titres - diluant ainsi les xeno-marchés dans la finance globale - et pour cela y être autorisées par voie de déréglementation. Le territoire, sans une réglementation spécifique, perd beaucoup de sa consistance économique. Sans différence réglementaire entre territoires, une variable importante présidant aux anciens arbitrages territoriaux des MN et des BMN a disparu. La déréglementation a décloisonné verticalement le marché financier mondial en faisant communiquer en permanence les marchés monétaires et financiers, les marchés des changes et les marchés des titres et des options, amenuisant la distinction entre les monnaies (les crédits en devises) et les actifs (les titres). Le décloisonnement est aussi horizontal en mettant en communication continue les espaces financiers nationaux. La formation de ce marché global des capitaux, fonctionnant en continu autour de la planète, est due à deux autres facteurs : le développement de la télématique permettant d'opérer sur des titres dématérialisés instantanément sur toutes les places financières internationales localisées autour du globe, à coûts de transaction réduits (grâce aux réseaux télématiques de transmission des données financières par satellites); la titrisation et les innovations financières issues de la crise de l'endettement international. L'ouverture des marchés boursiers aux investisseurs étrangers a renforcé le rôle de plaque tournante des places financières internationales dans le mouvement mondial des capitaux, de l'IDE et de l'acquisition de firmes étrangères. Ceci vaut aussi pour les bourses qui émergent dans une trentaine de PVD (dont Corée, Turquie, Mexique, Taïwan, Brésil, Thaïlande, Argentine, Malaisie, Indonésie, Chine) et d'économies en transition (Hongrie, Pologne, République tchèque, Russie, Ukraine, pays baltes). La taille des marchés émergents était de plus de 13 milliards de dollars en 1993 (5,7 milliards de dollars en 1992 pour le seul marché coréen). Localisées autour du globe, dans différents fuseaux horaires, les places financières internationales permettent aux BMN d'opérer 24 heures sur 24 sur le marché planétaire des changes et des capitaux; elles peuvent spéculer sur le mark sur les places asiatiques ou américaines pendant que les marchés européens sont fermés et que les banquiers centraux (Bundesbank) dorment. Les BMN ont réagi, après 1982, à la crise de l'endettement international et à leurs ratios de solvabilité détériorés en développant le hors-bilan, pour soustraire du bilan les créances douteuses, en les plaçant sous forme de titres (titrisation) auprès d'emprunteurs finals (désintermédiation) à l'aide d'innovations financières, facilitées par la déréglementation. Dans les conversions de dette, la sécurité de l'opération vient souvent de ce que le principal est garanti par des obligations à coupon zéro (l'une des innovations) libellées dans la même devise que la dette. La liste des nouveaux produits financiers s'est vite enrichie : échanges de dettes (swaps), euro-notes (titres négociables à court terme émis directement par les entreprises), xeno-obligations à taux d'intérêt variable ou révisable, obligations 
convertibles en actions, obligations à coupon zéro (le détenteur touche les intérêts en une seule fois lors du remboursement), obligations prorogeables, facilités d'émission d'effets (garantie accordée par une ou des BMN à l'absorption par le marché de titres à court terme émis par l'emprunteur), facilités à options multiples. Une deuxième vague d'innovations a donné naissance aux produits dérivés (plus de 10000 milliards de notionnel en décembre 1992) : options d'achat de devises, stellage (straddle) ou achat simultané d'une option d'achat (call) et d'une option de vente (put) de devises, contrats à terme (futures) d'achat de devises, lookback options où le prix des devises est déterminé à l'échéance, options asiatiques ou options sur moyennes, forward rate agreements qui fixent à l'avance le taux d'intérêt d'un prêt futur, options de taux d'intérêt, contrats cap et floor garantissant le versement de la différence entre le taux d'intérêt du marché à l'échéance et un taux (plafond ou plancher) garanti, collar (combinaison de cap et de floor), tunnels (combinaisons simples d'options de change), swaps de taux d'intérêt, swaptions ou swaps sur les options. Tous ces produits ont supplanté ceux des xeno-marchés traditionnels. La vitesse de circulation des nouveaux produits financiers est telle que les opérateurs ne peuvent plus vérifier les garanties offertes par le dernier emprunteur, ce qui augmente le risque de système (de défaillances en chaîne des opérateurs). Leur usage permet de passer d'un compartiment à l'autre du marché financier et s'étend avec la concurrence entre les BMN exacerbée par la globalisation. Derrière les produits financiers, il y a bien entendu le capital, chaque jour plus ubiquiste ${ }^{48}$, qui s'accumule sur la base d'une épargne mondiale mobilisée en tout point du globe et circulant facilement de territoire à territoire.

Conclusion : le " mondial sans territoire " est-il régulable? Dans la mesure où il est admis, par l'ONU ${ }^{49}$, que l'activité économique mondiale est devenue trop vulnérable au comportement des marchés de capitaux internationaux et à la faillite d'une grande BMN ou d'une société financière $\mathrm{MN}$, et qu'elle ne peut plus être régulée sur des bases territoriales nationales, un problème se pose. Une situation de défaut peut se propager à toute la chaîne des opérateurs sur le marché interbancaire. Les marchés financiers internationaux manquent d'un prêteur en dernier ressort ${ }^{50}$ qui rendrait la défaillance des BMN impossible. Certains envisagent de re-réglementer les mouvements internationaux de capitaux ou de taxer les opérations financières pour décourager les transactions purement spéculatives. De telles mesures paraissent inadmissibles aux MN et aux BMN et ont peu de chance d'être adoptées. Une alternative est d'instaurer de nouvelles règles prudentielles - tel le ratio Cooke, rapport minimal entre fonds propres et les risques des BMN, introduit en 1993 - afin de limiter les risques pris sur le marché financier planétaire: l'activité des BMN devrait être supervisée par les autorités monétaires du pays d'origine qui auront le droit de demander des informations sur l'activité des filiales étrangères. Mais ce dispositif n'affecte en rien les opérations financières des $\mathrm{MN}$ et a fortiori leurs activités non financières. Cette tentative de revaloriser le territoire national face à la mondialisation du capital suffira-t-elle face à une sorte "d'internationale de l'optimisation des rendements financiers nets " ${ }_{51}$, MN et BMN en tête, qui maximise ses gains spéculatifs et minimise son prélèvement fiscal ? Les années à venir n'invitent pas à un excès d'optimisme si la tendance lourde à la déterritorialisation croissante de l'activité des $\mathrm{MN}$ et des BMN, qui dure depuis près de trente ans, devait se prolonger comme étant un des fondements du capitalisme mondial. 


\section{NOTES}

1. C. Oman, Les nouvelles formes d'investissement dans les pays en développement, Paris, OCDE, 1984.

2. W. Andreff, Les multinationales, Paris, La Découverte, Coll. Repères, 2e éd., 1990.

3. W. Andreff, "Firmes multinationales et opinions publiques ", in L. Reboud (dir.), Les États dans le Dialogue international au XXIe siècle, Grenoble, CUREI, 1992.

4. Y. Bertrand, "L'exterritorialisation des entreprises : aspects récents du comportement des entreprises d'origine bretonne ", in M. Humbert (dir.), L'Europe face aux mutations mondiales, Paris, Economica, 1993.

5. Y. Bertrand, op. cit.

6. Y. Bertrand, op. cit.

7. C.A. Michalet, Le capitalisme mondial, 2e éd., Paris, PUF, 1985.

8. C.A. Michalet, op. cit.

9. M.E. Porter (ed.), Competition in Global Industries, Harvard Business School Press, 1986.

10. W. Andreff, “Firmes Multinationales et opinions publiques ”, op. cit.

11. P. Jacquemot, La firme multinationale : Une introduction économique, Paris, Economica, 1990.

12. B. Lassudrie-Duchêne, " Décomposition internationale des processus productifs et autonomie nationale ", in $\mathrm{H}$. Bourguinat (dir.), Internationalisation et autonomie de décision, Paris, Economica, 1982.

13. W. Andreff, Profits et structures du capitalisme mondial, Paris, Calmann-Lévy, 1976.

14. ONU, World Investment Report 1993 : Transnational Corporations and Integrated International Production, New York, UNCTAD, UN, 1993.

15. W. Andreff, Les multinationales hors la crise, Le Sycomore, 1982.

16. W. Andreff, Les multinationales hors la crise, Le Sycomore, 1982.

17. P. Jacquemot, op. cit.

18. G. Lafay, C. Herzog, " La création des avantages comparatifs dans les activités de haute technologie ", Revue d'Economie Industrielle, n 55, 1991.

19. M. Delapierre, L.K. Mytelka, " Décomposition, recomposition des oligopoles ", Economies et Société, série p. 31, 1988.

20. E.M. Mouhoud, Changement technique et division internationale du travail, Paris, Economica, 1992.

21. E.M. Mouhoud, op. cit.

22. J.H. Dunning, The Globalization of Business, Routledge, 1993.

23. J. Howells, M. Wood, The Globalisation of Production and Technology, Belhaven Press, 1993.

24. Lafay, Herzog, op. cit.

25. C.A. Michalet, "Les accords interfirmes internationaux : un cadre pour l'analyse ", dans R. Arena et alii, Traité d'économie industrielle, Paris, Economica, 1988. ; J.H. Dunning, op. cit.

26. M. Delapierre, C.A. Michalet, "Vers un changement des structures des multinationales : le principe d'internalisation en question ", Revue d'Economie Industrielle, $\mathrm{n}^{\circ} 47,1989$.

27. J.H. Dunning, op. cit. 
28. ONU, World Investment Report 1992 : Transnational Corporations as Engines of Growth, New York, UNCTC, UN, 1992.

29. M. Delapierre, “Les accords inter-entreprises, partage ou partenariat? ", Revue d'Economie Industrielle, $\mathrm{n}^{\circ}$ 55, 1991.

30. C.A. Michalet, op. cit.

31. F. Chesnais, La mondialisation du capital, Paris, Syros, 1994.

32. K. Ohmae, Beyond National Borders. Reflections on Japan and the World, Dow Jones - Irwin, 1987.

33. OCDE, Trade, Investment and Technology in the 1990s, Paris, 1991.

34. ONU, World Investment Report. Transnational Corporations, Employment and the Workplace, New York, UNCTAD, UN, 1994.

35. M.E. Porter, L'avantage concurrentiel des nations, InterEditions, 1993.

36. J. Savary, “European integration, globalisation and industrial location in Europe ”, in H. Cox, Clegg J., Ietto-Gillies G. (eds.), The Growth of Global Business, Routledge, 1993. 37. J.H. Dunning, Explaining International Production, Harper Collins, 1988.

38. L. Walter, "The role of mergers and acquisitions in foreign direct investment ", in L. Oxelheim (ed.), The Global Race for Foreign Direct Investment. Prospects for the Future, Springer-Verlag, 1993.

39. Howells, Wood, op. cit.

40. ONU, Transnational Corporations in World Development. Trends and Prospects, New York, UNCTC (Centre on Transnational Corporations), UN, 1988.

41. R.E. Caves, A. More, "Intrafirm royalties in the process of expansion of US multinational enterprises ", in V.N. Balasubramanyam, D. Sapsford (eds)., The Economics of International Investment, Edward Elgar, 1994.

42. ONU, Transnational Corporations in World Development : Trends and prospects, op. cit. . La surfacturation des importations d'une filiale en provenance de la mère ou la sous-facturation des livraisons de la filiale à la mère permettent de transférer des fonds du pays hôte vers le pays d'origine. Ainsi les MN industrielles se diversifient vers les activités financières, se " financiarisent", et la frontière entre finance et industrie devient floue. Quant aux frontières des territoires nationaux ou locaux, ils sont presque totalement perméables à ces flux financiers, pour la plupart incontrôlables, contrôle d'ailleurs de moins en moins souhaité par des autorités nationales et locales séduites par les sirènes de la déréglementation. Il en résulte à la fois qu'une importante masse de liquidités circule régulièrement dans la $\mathrm{MN}$, mais entre pays, d'un territoire à l'autre, et qu'elle peut être mobilisée à tout moment par la MN pour intervenir sur les marchés financiers. L'objectif en est de se couvrir contre le risque de change ou de taux d'intérêt par le termaillage (leads and lags) qui consiste à modifier les délais de paiement des transactions commerciales et de cession de devises ; par l'endiguement (hedging) qui consiste à modifier les monnaies de facturation au comptant ou à terme, de façon à réduire le risque ou à tirer des profits de la spéculation sur les fluctuations de change ; par l'avance de devises de la mère aux filiales. Les MN exploitent les changements de parité quand elles ne les provoquent pas. Les MN peuvent aussi réduire le float (ou devises en transit) - la masse de fonds circulant entre filiales - pour éviter de rester sur des monnaies faibles, ou compenser (netting) les créances et les engagements de même échéance entre mère et filiales, soustrayant les montants compensés au risque de change[[H. Bourguinat, Finance internationale, Paris, PUF, Coll. Thémis, 1992. 
43. D. Plihon, “ Les mécomptes de la globalisation financière ”, Alternatives économiques, $\mathrm{n}^{\circ} 20,1994$.

44. D. Plihon, op. cit.

45. H. Bourguinat, op. cit.

46. H. Bourguinat, " Renégocier Bretton Woods ? La spéculation internationale comme variable trouble-fête ", in M. Rainelli (dir.), La négociation commerciale et financière internationale, Economica (à paraître).

47. ONU, op. cit.

48. H. Bourguinat, Finances internationales, op. cit.

49. ONU.

50. M. Aglietta, A. Brender, V. Coudert, Globalisation financière : l'aventure obligée, CEPII, Paris, Economica, 1990.

51. H. Bourguinat, “ Renégocier Bretton Woods ”, op. cit.

INDEX

Mots-clés : économie politique, entrepreunariat, territoire(s) et territorialité, Mondialisation 